\title{
Daily activity level improvement with antidepressant medications predicts long-term clinical outcomes in outpatients with major depressive disorder
}

\author{
This article was published in the following Dove Press journal: \\ Neuropsychiatric Disease and Treatment \\ 15 March 2017 \\ Number of times this article has been viewed
}

\author{
Manish K Jha' \\ Raymond B Teer ${ }^{2}$ \\ Abu Minhajuddin ${ }^{3}$ \\ Tracy L Greer' \\ A John Rush ${ }^{4}$ \\ Madhukar H Trivedi \\ 'Center for Depression Research \\ and Clinical Care, University of \\ Texas Southwestern Medical Center, \\ Dallas, ${ }^{2}$ University of Texas, Austin, \\ ${ }^{3}$ Department of Clinical Sciences, \\ University of Texas Southwestern \\ Medical Center, Dallas, TX, USA; \\ ${ }^{4}$ Duke-NUS, Singapore, Singapore
}

\begin{abstract}
Background: Major depressive disorder (MDD) significantly impacts performance of both work- and nonwork-related routine daily activities. We have shown that work productivity is significantly impaired in employed MDD patients, but the extent of impairments in nonworkrelated routine activities and its association with antidepressant treatment outcomes has not been established.

Materials and methods: Activity impairment was measured using the sixth item of Work Productivity and Activity Impairment Scale in the Combining Medications to Enhance Depression Outcomes (CO-MED) trial ( $\mathrm{n}=665$ ). Published norms were used to define activity impairment levels. The relationship between activity impairment and baseline sociodemographic and clinical characteristics was evaluated along with changes in activity impairment and its relationship with other clinical outcomes such as symptom severity, function, and side effect burden. Remission status at 3 and 7 months was predicted based on week 6 activity impairment level.

Results: Higher psychosocial and cognitive impairments and greater number of comorbid medical conditions were associated with greater activity impairment at baseline. Proportion of participants with severe activity impairment declined from $47.6 \%$ at baseline to $18.7 \%$ at 3 months, while mean activity impairment decreased from 57.1 at baseline to 32.8 at 3 months. During course of treatment, levels of activity impairment correlated most strongly with psychosocial function among measures of symptom severity, function, quality of life, and side effect burden. No or minimal activity impairment at week 6 was associated with two to three times higher rates of remission at 3 and 7 months as compared to moderate or severe activity impairment levels even after controlling for remission status at week 6 and select baseline variables.

Conclusion: Depressed patients have high levels of nonwork-related activity impairment at baseline that improves significantly with treatment and independently predicts long-term clinical outcomes. Brief systematic assessment of activity impairment during the course of antidepressant treatment can help inform clinical decision-making.
\end{abstract}

Keywords: depression, activity impairment, predictors, functional recovery, productivity

\section{Introduction}

Major depressive disorder (MDD) affects one in six adults in the United States during their lifetime. ${ }^{1,2}$ Depression is the second leading cause of disability globally. ${ }^{3}$ The diagnostic criteria of MDD include a change in functioning from baseline. ${ }^{4}$ However, the types of functional impairments due to MDD vary widely. ${ }^{4}$ Impairments due to depression affect multiple facets of life, including work productivity, ${ }^{5,6}$ social functions, ${ }^{7,8}$ 
sexual satisfaction, ${ }^{9}$ life satisfaction, ${ }^{10,11}$ interpersonal function, ${ }^{12}$ quality of life, ${ }^{13}$ and overall health. ${ }^{14}$ Improvement with antidepressant treatment is not just restricted to change in depression severity. ${ }^{15-17}$ In a previous report of employed MDD patients, we have found that work productivity is significantly impaired, improves early with treatment independent of depression severity, and is associated with better longer term outcomes. ${ }^{18}$ However, this assessment of work productivity improvement is restricted to those who are employed. Being unemployed prior to treatment initiation has reportedly been associated with worse antidepressant treatment outcomes. ${ }^{5,19}$ Thus, it is important to identify the extent of productivity impairment in both employed and unemployed depressed patients and test its association with subsequent clinical outcomes.

As a productivity outcome, previous reports have identified impairments in the ability of depressed patients to perform nonwork-related routine day-to-day activities (or activity impairment). ${ }^{20,21}$ Antidepressant treatment results in reduced activity impairment. ${ }^{22}$ However, the clinical significance of these improvements is unclear. In contrast to the consensus guidelines to evaluate change in depression severity with treatment, ${ }^{23}$ there are no similar easy-to-interpret recommendations for measuring or conceptualizing changes in activity impairment. While antidepressant medications are superior to placebo, ${ }^{22}$ whether individual medications or their combinations differentially improve activity impairment is unclear. Similarly, while reduced depression severity is associated with improvement in activity impairment, ${ }^{22}$ the relationship of activity impairment with other aspects of functioning as well as treatment-emergent side effects in depressed patients continues to be unknown.

This report focuses on depressed outpatients. We use the term "activity" to refer to nonwork-related activity (eg, daily chores and responsibilities). The report addresses the following specific questions:

1. What baseline clinical and sociodemographic characteristics are associated with greater activity impairment?

2. Does activity impairment improve overall with acuteand continuation-phase antidepressant medication treatment?

3. Do antidepressant medications differ in their effect on activity impairment?

4. Which baseline variables predict subsequent improvement in activity impairment?

5. Does activity impairment relate to other clinical outcomes such as symptom severity, function, and side effect burden during course of antidepressant treatment?
6. Does activity impairment level at week 6 predict longer term treatment outcomes even after controlling for remission status and select baseline sociodemographic and clinical variables?

This is an unplanned secondary analysis of data from Combining Medications to Enhance Depression Outcomes (CO-MED) trial, ${ }^{24}$ which included three treatment arms (escitalopram plus placebo, bupropion plus escitalopram, and venlafaxine plus mirtazapine) in the acute-phase treatment that lasted 3 months. Continuation-phase data were collected for another 4 months thereafter. In the analytic cohort $(n=665)$, we evaluated activity impairment prior to treatment initiation and its association with baseline characteristics. We used the method proposed by Vittengl et $\mathrm{al}^{25}$ that "severe impairment" in functioning can be defined based on assumption that $10 \%$ of a community sample suffers from impaired function due to mental illness and hence operationalized as beyond 1.28 standard deviation (SD) from the mean of best available community norms. Hence, we used findings from the large-scale National Health and Wellness Survey (NHWS, n=75,000) to classify depressed patients in the following three groups: no or minimal level of activity impairment (less than mean of NHWS), moderate activity impairment (equal to or greater than mean and less than or equal to mean plus 1.28 SD of NHWS), and severe activity impairment (greater than mean plus 1.28 SD of NHWS). We then evaluated the overall improvement in activity impairment with treatment and whether the three treatment arms differed in their effect on activity impairment. We also evaluated the relationship of levels of activity impairment with levels of symptom severity, functional outcomes (psychosocial function, cognitive and physical function, and quality of life), and treatment-associated side effects.

\section{Materials and methods}

\section{Study overview and participants}

All participants in the CO-MED trial $(n=665)$ constitute the analytic sample of this report. The CO-MED trial (NCT00590863) ${ }^{24}$ was approved by the institutional review boards at the University of Texas Southwestern Medical Center at Dallas, the University of Pittsburgh Data Coordinating Center, and each participating regional center and clinical site, and it was monitored by an independent data safety and monitoring board. A separate approval for this specific project was not needed by the institutional review boards, as it is based on the secondary outcomes which were collected as part of CO-MED trial. The primary paper by Rush et al, ${ }^{24}$ mentions the scale but did not report on activity impairment as an outcome. All participants provided written informed consent prior to completing any study related procedures, including the Work 
Productivity and Activity Impairment Scale. Participants from six primary and nine psychiatric care sites had nonpsychotic chronic (current episode exceeded 2 years) or recurrent depression with current episode $\geq 2$ months and a baseline 17-item Hamilton Rating Scale (HRSD17) $\geq 16$. Rush et al $^{24}$ have previously reported detailed inclusion and exclusion criteria.

After enrollment into the trial and stratification by clinical sites, participants were assigned to one of the following three treatment arms in a 1:1:1 ratio: 1) escitalopram plus placebo (selective serotonin reuptake inhibitor [SSRI] monotherapy), 2) sustained-release (SR) bupropion plus escitalopram (bupropion-SSRI combination), and 3) extended-release (XR) venlafaxine plus mirtazapine (venlafaxine-mirtazapine combination). Participants were seen for study visits at baseline and at weeks $1,2,4,6,8,10$, and 12 for acute phase and weeks $16,20,24$, and 28 for continuation phase. During the first 8 weeks, study physicians used measurement-based care ${ }^{26}$ at each visit to make antidepressant dosage adjustments based on the scores of the Quick Inventory of Depressive Symptomatology Clinician-rated version (QIDS-C) ${ }^{27}$ scale and the Frequency, Intensity, and Burden of Side Effects Rating scale. ${ }^{28}$ Participants were allowed to proceed to the continuation phase (beyond 3 months) only if they 1) had received an acceptable benefit (defined as QIDS-C score of 9 or less by 3 months) or 2) had reached a score of 10-13 on QIDS-C and both the study physician and the participant decided to continue treatment because of substantial benefit. As previously reported, 479 participants were enrolled in the continuation phase. ${ }^{24}$

\section{Assessments}

The following measures were obtained at baseline and each subsequent study visit of acute phase (weeks 1, 2, 4, 6, 8, 10, and 12) and continuation phase (weeks 16, 20, 24, and 28).

Work productivity and impairment (WPAI): the WPAI self-report has good construct validity and test-retest reliability. ${ }^{29}$ The sixth item on the scale was used to measure nonwork-related impairment in regular daily activities "such as work around the house, shopping, childcare, exercising, studying, etc." (or activity impairment). The activity impairment item (\#6 of WPAI) is rated on a scale of $0-10$, where 0 indicates no impairment and 10 indicates impairment that completely prevents participants from doing daily activities. Using the scoring guide by Reilly, ${ }^{30}$ percentage of activity impairment was calculated using the formula: $10 \times($ item 6 ), with higher scores reflecting greater impairment and comprised the primary outcome measure for the current report. In addition to this score, we categorized participants on the basis of their activity impairment using the method described by Vittengl et al. ${ }^{25}$ In the large-scale NHWS $(n=75,000)$, the mean (SD) activity impairment of noncaregiver respondents who can be considered as best available community norm $(\mathrm{n}=69,224)$ was reported as $25.22(\mathrm{SD}=29.32) .{ }^{31}$ Using the Vittengl et $\mathrm{al}^{25}$ formula: (mean) $+(1.28 \times \mathrm{SD})$, we estimated the threshold for severe activity impairment as scores greater than 62.75. Additionally, we established a threshold of no or minimal activity impairment as less than the mean of community norm. Hence, we categorized participants in the following three groups: no or minimal activity impairment (activity impairment score $<25.22$ ), moderate activity impairment (activity impairment score $\geq 25.22$ and $\leq 62.75$ ), and severe impairment (activity impairment score $>62.75$ ).

QIDS-C and self-report (QIDS-SR): the total score of QIDS-C and QIDS-SR (range of 0-27) is based on the nine criterion symptom domains out of the 16 items, each of which is scored from 0 to $3 .{ }^{27}$ The Pearson moment correlations were 0.86 between QIDS-SR and HRSD17 and 0.93 between QIDS-C and HRSD17 in a previous report. ${ }^{32}$ The Cronbach's $\alpha$ of QIDS-SR and QIDS-C has ranged from 0.86 to 0.87 in previous reports. ${ }^{27,33}$ The QIDS-SR served as the primary measure of depressive symptoms. The QIDS-C was completed by the clinician to monitor symptom changes and guide treatment decisions.

Work and social adjustment scale (WSAS): this five-item self-report measure of functional impairment has been used to study the treatment of depression and anxiety. ${ }^{34}$ Each item is rated on a $0-8$ scale, with 0 indicating no impairment and 8 indicating very severe impairment, and a total score range of $0-40$. The WSAS scores above 10 suggest clinically meaningful functional impairment. Cronbach's $\alpha$ measure of internal consistency of WSAS range from 0.80 to 0.94 , with test-retest reliability of 0.73 . The WSAS also has good convergent and discriminant validity and is sensitive to patient differences in severity, as well as treatment-related change. ${ }^{34,35}$

Quality of Life Inventory (QOLI): the 32 QOLI items assess 16 domains of life. ${ }^{36}$ After a brief description of each specific domain (such as "HEALTH is being physically fit, not sick, and without pain or disability"), an initial question asks about the importance of that specific domain to happiness; response options are "not important," "important," and "extremely important." A subsequent question then asks about satisfaction with that specific domain and the six options range from -3 (very dissatisfied) to +3 (very satisfied). This allows scoring of QOLI to incorporate the importance of each domain into the overall well-being assessment for a given individual. The correlation coefficients of QOLI and other measures of life satisfaction (eg, Satisfaction with Life Scale and Quality of Life Index) range from 0.56 to $0.71 .^{36}$ The Cronbach's $\alpha$ of QOLI ranges from 0.79 to 
$0.85 .^{36,37}$ The predictive validity and sensitivity to treatment change of QOLI have been supported in multiple previous studies. $^{38}$

The Massachusetts General Hospital Cognitive and Physical Functioning Questionnaire (CPFQ): the total score of this seven-item self-report questionnaire is calculated by adding the following items: motivation/interest, wakefulness/ alertness, energy, ability to focus, ability to remember, ability to find words, and sharpness/mental acuity. The Pearson moment correlation between CPFQ and HRSD17 ranged from 0.24 to 0.29 in previous report. ${ }^{39}$ The Cronbach's $\alpha$ of CPFQ has ranged from 0.89 to 0.91 in previous reports. ${ }^{39,40}$

Frequency, Intensity, and Burden of Side Effect Rating (FIBSER) Scale: this commonly used side effect rating scale measures the frequency, intensity, and burden of side effects with three items rated on a scale of $0-6$ with higher numbers reflecting greater severity of side effects. ${ }^{28}$ The Cronbach's $\alpha$ of FIBSER ranged from 0.91 to 0.93 at different study visits (weeks 2, 4, 6, 9, 12, and 14) in a previous report. ${ }^{28}$ The sum of three items has been used as an overall score of FIBSER. ${ }^{41}$

\section{Statistical analyses}

We included all CO-MED trial participants $(n=665)$ as the analytic sample for this report. We used Chi-square test and analysis of variance using PROC GLM as the univariate analyses to test the association of activity impairment categories (no or minimal, moderate, and severe) with categorical and continuous baseline variables, respectively. We then included all variables with $P \leq 0.05$ in the abovementioned univariate analyses in a logistic regression analysis with baseline activity impairment category as the dependent variable. We used separate repeated measures mixed model analyses to test for change in activity impairment during the acute and continuation phases of CO-MED trial. To test if antidepressant medication combinations differed from SSRI monotherapy in improvement of activity impairment, we included treatment arm as a covariate in abovementioned mixed model analyses along with baseline levels of depression severity, activity impairment, and significant baseline variables from the abovementioned multivariate analyses. In separate mixed model analyses, we excluded baseline measures of psychosocial function as well as cognitive function to test the predictive effect of other baseline variables on changes in activity impairment during acute and continuation phases. For both acute and continuation phases of CO-MED, we separately calculated correlation coefficient between the activity impairment and depressive symptoms (nine domains as well as total score of QIDS-SR), psychosocial functioning
(WSAS), quality of life (QOLI), cognitive and physical functioning (CPFQ), and side effects (FIBSER) using PROC MIXED as implemented in $\mathrm{SAS}^{42}$ as we had repeated observations for each subject. ${ }^{43}$ To test long-term clinical outcomes based on week 6 activity impairment categories, we used separate logistic regression analyses to predict remission status at 3 and 7 months. We also conducted multivariate logistic regression analyses to account for remission status at week 6 as well as baseline variables that have been associated with poor treatment outcomes (such as employment status, baseline depression severity, and presence of anxious features) as well as those variables identified to be independently associated with activity impairment at baseline. We used separate repeated measures mixed model analyses to test for change in depression severity (QIDS-SR) based on activity impairment category at week 6 over the remaining course of acute phase (week 6 to 3 months) and continuation phase (3-7 months) before and after controlling for remission status at week 6 and abovementioned baseline variables.

To conduct an extensive search of baseline clinical and sociodemographic features that are associated with activity impairment in depressed patients, we included the following variables collected in the CO-MED trial: sex, age, body mass index, education (less than 12, 12-15, and 16+ years of schooling), employment status, monthly income $(<\$ 2,000$, $\$ 2,000$ to $\$ 4,000$, and greater than $\$ 4,000$ ), race (white, black, other), Hispanic ethnicity, presence of anxious, atypical, or melancholic features at baseline, onset of depressive symptoms before age of 18 , presence of suicidal ideations or anhedonia, lifetime history of suicide attempt, presence of chronic or recurrent depression, duration of current episode, number of comorbid general medical conditions, number of comorbid psychiatric conditions, and levels of depressive symptom severity, cognitive function, psychosocial function, and quality of life.

We set the level of significance at 0.05 and used SAS 9.3 (SAS Inc., Cary, NC, USA) for all analyses.

\section{Results}

The clinical and sociodemographic features of participants according to categories of activity impairment at baseline and the results of univariate analyses are presented in Table 1.

\section{What baseline clinical and sociodemographic characteristics are associated with greater activity impairment?}

In multivariate analyses of the baseline variables listed in Table 1 with $P \leq 0.05$, we found that psychosocial function as measured by the WSAS, self-reported cognitive and 
Table I Baseline sociodemographic and clinical characteristics of CO-MED trial participants according to baseline activity impairment

\begin{tabular}{|c|c|c|c|c|c|c|c|c|c|c|}
\hline \multirow{3}{*}{$\frac{\text { Number }}{\text { Categorical variables }}$} & \multirow{2}{*}{\multicolumn{2}{|c|}{$\begin{array}{l}\text { Total } \\
664^{a}\end{array}$}} & \multirow{2}{*}{\multicolumn{2}{|c|}{$\begin{array}{l}\text { No or } \\
\text { minimal } \\
\text { impairment } \\
140\end{array}$}} & \multirow{2}{*}{\multicolumn{2}{|c|}{$\begin{array}{l}\text { Moderate } \\
\text { impairment } \\
208\end{array}$}} & \multirow{2}{*}{\multicolumn{2}{|c|}{$\begin{array}{l}\text { Severe } \\
\text { impairment } \\
316\end{array}$}} & \multirow[b]{3}{*}{ Chi-square (df) } & \multirow[b]{3}{*}{$P$-value } \\
\hline & & & & & & & & & & \\
\hline & $\mathbf{n}$ & $\%$ & $n$ & $\%$ & $\mathbf{n}$ & $\%$ & $\mathbf{n}$ & $\%$ & & \\
\hline Sex & & & & & & & & & $78.66(2)$ & 0.02 \\
\hline Male & 213 & 32.1 & 56 & 40.0 & 71 & 34.1 & 86 & 27.2 & & \\
\hline Female & 451 & 67.9 & 54 & 60.0 & 137 & 65.9 & 230 & 72.8 & & \\
\hline Race & & & & & & & & & $0.80(4)$ & 0.94 \\
\hline White & 425 & 64.0 & 91 & 65.0 & 128 & 61.5 & 206 & 65.2 & & \\
\hline Black & 176 & 26.5 & 36 & 25.7 & 59 & 28.4 & 81 & 25.6 & & \\
\hline Other & 63 & 9.5 & 13 & 9.3 & 21 & 10.1 & 29 & 9.2 & & \\
\hline Monthly income & & & & & & & & & $6.53(4)$ & 0.16 \\
\hline$<\$ 2,000$ & 378 & 62.9 & 67 & 54.9 & 132 & 68.1 & 179 & 62.8 & & \\
\hline$\$ 2,000-\$ 4,000$ & 128 & 21.3 & 29 & 23.8 & 35 & 18.0 & 64 & 22.5 & & \\
\hline$>\$ 4,000$ & 95 & 15.8 & 26 & 21.3 & 27 & 13.9 & 42 & 14.7 & & \\
\hline Treatment arm & & & & & & & & & $7.94(2)$ & 0.09 \\
\hline SSRI monotherapy & 224 & 33.7 & 43 & 30.7 & 77 & 37.0 & 104 & 32.9 & & \\
\hline Bupropion plus SSRI & 220 & 33.1 & 58 & 41.4 & 67 & 32.2 & 95 & 30.1 & & \\
\hline Venlafaxine plus mirtazapine & 220 & 33.1 & 39 & 27.9 & 64 & 30.8 & 117 & 37.0 & & \\
\hline Education & & & & & & & & & I.II (4) & 0.89 \\
\hline$<12$ years & 98 & 15.3 & 17 & 12.8 & 33 & 16.3 & 48 & 15.7 & & \\
\hline $12-15$ years & 351 & 54.8 & 73 & 54.9 & 111 & 55.0 & 167 & 54.8 & & \\
\hline$>15$ years & 191 & 29.9 & 43 & 32.3 & 58 & 28.7 & 90 & 29.5 & & \\
\hline Hispanic ethnicity & 101 & 15.2 & 17 & 12.1 & 39 & 18.8 & 45 & 14.2 & $3.27(2)$ & 0.19 \\
\hline Employed at baseline & 331 & 49.8 & 81 & 57.9 & 109 & 52.4 & $14 \mid$ & 44.6 & $7.59(2)$ & 0.02 \\
\hline Suicidal ideations at baseline & 394 & 59.3 & 78 & 55.7 & 114 & 54.8 & 202 & 63.9 & $5.29(2)$ & 0.07 \\
\hline Onset of depression before age 18 & 296 & 44.7 & 72 & 51.4 & 78 & 37.7 & 146 & 46.4 & $7.04(2)$ & 0.03 \\
\hline Chronic depression & 368 & 55.6 & 73 & 52.1 & 128 & 61.8 & 167 & 53.0 & $4.79(2)$ & 0.09 \\
\hline Recurrent depression & 516 & 77.9 & 115 & 82.1 & $|5|$ & 73.0 & 250 & 79.4 & $4.81(2)$ & 0.09 \\
\hline Lifetime history of suicide attempt & 59 & 9.2 & 14 & 10.4 & 18 & 8.9 & 27 & 8.9 & $0.29(2)$ & 0.87 \\
\hline Presence of melancholic features & 227 & 34.2 & 35 & 25.0 & 61 & 29.3 & $|3|$ & 41.46 & $14.86(2)$ & $<0.001$ \\
\hline Presence of atypical features & 123 & 18.5 & 27 & 19.3 & 37 & 17.8 & 59 & 18.7 & $0.13(2)$ & 0.94 \\
\hline Presence of anhedonia & 318 & 47.9 & 54 & 38.6 & 86 & $4 I .4$ & 178 & 56.3 & $17.46(2)$ & $<0.001$ \\
\hline Presence of anxious features & 496 & 74.9 & 95 & 67.9 & 150 & 72.5 & 251 & 79.7 & $8.18(2)$ & 0.02 \\
\hline Continuous variables & Mean & SD & Mean & SD & Mean & SD & Mean & SD & F-value (df) & $P$-value \\
\hline Mean age (years) & 42.7 & 13.0 & 41.9 & 13.3 & 43.6 & 13.3 & 42.5 & 12.7 & $0.77(2,661)$ & 0.46 \\
\hline Weight (pounds) & 194.1 & 59.2 & 195.2 & 54.5 & 195.8 & 59.3 & 192.5 & 61.2 & $0.23(2,660)$ & 0.80 \\
\hline BMI & 31.0 & 8.8 & 30.5 & 7.5 & 31.3 & 9.1 & 31.1 & 9.2 & $0.36(2,658)$ & 0.70 \\
\hline Mean QIDS-SR & 15.5 & 4.3 & 14.1 & 5.0 & 14.2 & 4.3 & 16.9 & 3.6 & $38.16(2,66 I)$ & $<0.001$ \\
\hline Mean WSAS & 26.9 & 8.8 & 21.6 & 9.5 & 24.4 & 7.9 & 30.9 & 7.1 & $82.09(2,660)$ & $<0.001$ \\
\hline Mean QOLI & 21.0 & 14.0 & 24.0 & 13.9 & 23.9 & 13.6 & 17.1 & 13.6 & $17.36(2,66 I)$ & $<0.001$ \\
\hline Mean CPFQ & 27.6 & 5.9 & 25.7 & 5.9 & 25.9 & 5.4 & 29.6 & 5.5 & $39.74(2,66 I)$ & $<0.001$ \\
\hline Activity impairment & 57.1 & 32.0 & 7.4 & 8.5 & 48.2 & 10.6 & 84.9 & 10.7 & $2,880.38(2,66 I)$ & $<0.001$ \\
\hline Number of general medical conditions & 1.9 & 1.6 & 1.4 & 1.3 & 2.1 & 1.6 & 2.1 & 1.6 & $13.67(2,661)$ & $<0.001$ \\
\hline Number of comorbid psychiatric conditions & 1.3 & 1.6 & 1.0 & 0.9 & 1.2 & 1.6 & 1.5 & 1.7 & $5.70(2,660)$ & 0.004 \\
\hline Duration of current episode (weeks) & 61.7 & 104.8 & 61.9 & 108.8 & 63.9 & 103.8 & 60.3 & 104.0 & $0.07(2,659)$ & 0.68 \\
\hline
\end{tabular}

Notes: No or minimal, moderate, and severe activity impairment were defined as scores of activity impairment $<25.22,25.22-62.75$, and $>62.75$ based on the mean and standard deviation of activity impairment reported in the 2009 National Health and Wellness Survey. ${ }^{31}$ activity impairment information at baseline was missing for one participant, thus this number differs from the total number of participants $(n=665)$ in CO-MED trial (NCT00590863).

Abbreviations: CO-MED, Combining Medications to Enhance Depression Outcomes; SSRI, selective serotonin reuptake inhibitor; SD, standard deviation; df, degrees of freedom; BMI, body mass index; QIDS-SR, Quick Inventory of Depressive Symptomatology, self-report; WSAS, Work and Social Adjustment Scale; QOLI, Quality of Life Inventory; CPFQ, Cognitive and Physical Functioning Questionnaire.

physical function as measured by CPFQ, and number of general medical conditions were significantly associated with activity impairment. Based on the multivariate analyses, we found that participants with higher scores on WSAS were more likely to have moderate (odds ratio $[\mathrm{OR}]=1.05$,
$95 \%$ confidence interval $[\mathrm{CI}]=1.02,1.08)$ and severe $(\mathrm{OR}=1.14,95 \% \mathrm{CI}=1.10,1.17)$ activity impairment than no or minimal activity impairment. Participants with higher scores on CPFQ were more likely to have severe activity impairment $(\mathrm{OR}=1.06,95 \% \mathrm{CI}=1.01,1.12)$. Higher number 
of comorbid general medical conditions was associated with moderate $(\mathrm{OR}=1.46,95 \% \mathrm{CI}=1.23,1.74)$ as well as severe activity impairment $(\mathrm{OR}=1.45,95 \% \mathrm{CI}=1.21,1.73)$. We found no significant association of activity impairment categories with other baseline variables with $P \leq 0.05$ in univariate analyses: depression severity $\left(\chi^{2}=3.24, d f=2\right.$, $P=0.20)$, quality of life $\left(\chi^{2}=1.87, d f=2, P=0.39\right)$, treatment $\operatorname{arm}\left(\chi^{2}=3.66, d f=4, P=0.16\right), \operatorname{sex}\left(\chi^{2}=3.75, d f=2, P=0.15\right)$, baseline suicidal ideation $\left(\chi^{2}=0.55, d f=2, P=0.76\right)$, employment status $\left(\chi^{2}=0.86, d f=2, P=0.65\right)$, number of comorbid psychiatric disorders $\left(\chi^{2}=0.94, d f=2, P=0.62\right)$, presence of anxious features $\left(\chi^{2}=0.68, d f=2, P=0.71\right)$, or anhedonia $\left(\chi^{2}=0.06, d f=2, P=0.97\right)$.

Does activity impairment improve overall with acute- and continuation-phase antidepressant medication treatment?

Yes. During acute phase, activity impairment reduced significantly $(F=69.17, d f=7,3,528, P<0.0001)$ from baseline (least squares $[\mathrm{LS}]$ mean $=57.07$; standard error $[\mathrm{SE}]=1.22$ ) to 3 months (LS means=32.75; SE=1.41) in unadjusted mixed model analyses. Proportion of participants with severe activity impairment reduced from $47.6 \%$ at baseline to $25.3 \%$ at week 6 and $18.4 \%$ at 3 months (Figure 1). The improvement with time in activity impairment continued to be significant ( $F=32.43, d f=6,2,894, P<0.0001)$ even after controlling for baseline activity impairment, cognitive and physical function, psychosocial function, and number of general medical conditions. During continuation phase, the change in activity impairment was not statistically significant $(F=1.69, d f=4$, $1,565, P=0.15)$.

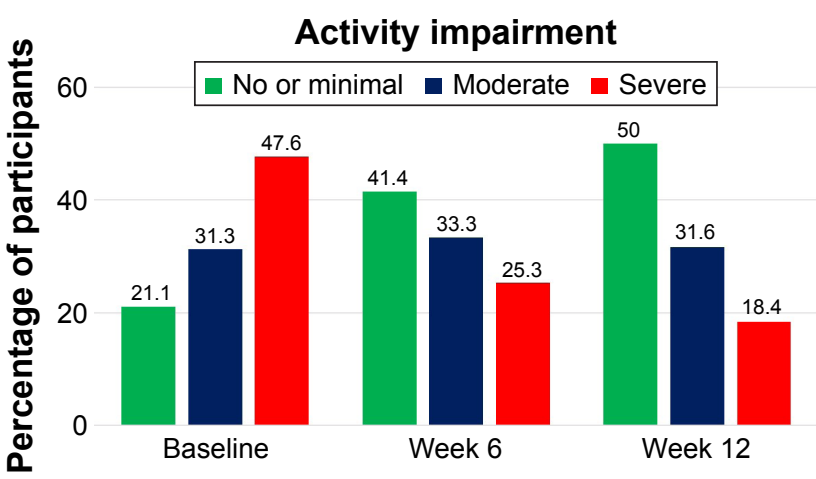

Figure I Impairment in ability to perform routine day-to-day activities during acute phase of CO-MED trial (NCT00590863).

Note: No or minimal, moderate, and severe activity impairment were defined as scores of activity impairment $<25.22,25.22-62.75$, and $>62.75$ based on the mean and standard deviation of activity impairment reported in the 2009 National Health and Wellness Survey. ${ }^{31}$

Abbreviation: CO-MED, Combining Medications to Enhance Depression Outcomes.
Do antidepressant medications differ in their effect on activity impairment?

No. The three treatment arms did not differ in rate of change of activity impairment during both acute $(F=0.34, d f=2$, $615, P=0.71)$ and continuation phases $(F=0.16, d f=2,468$, $P=0.85$, Figure 2 ).

\section{Which baseline variables predict subsequent} improvement in activity impairment?

In multivariate mixed model analyses, we found that during both acute and continuation phases, higher baseline levels of activity impairment (acute phase: estimate [est.] $=0.25, \mathrm{SE}=0.03$, $t=8.20, d f=607, P<0.001$; continuation phase: est. $=0.20$, $\mathrm{SE}=0.04, t=5.22, d f=460, P<0.001)$, cognitive and physical function impairment (acute phase: est. $=0.62, \mathrm{SE}=0.17, t=3.74$, $d f=620, P<0.001$; continuation phase: est $=0.42, \mathrm{SE}=0.21$, $t=2.01, d f=465, P=0.05)$, psychosocial function impairment (acute phase: est. $=0.50, \mathrm{SE}=0.12, t=4.26, d f=619, P=<0.001$; continuation phase: est. $=-0.29, \mathrm{SE}=0.15, t=-0.52, d f=462$, $P=0.05$ ), and higher number of comorbid general medical conditions (acute phase: est. $=3.67, \mathrm{SE}=0.58, t=6.37, d f=599$, $P<0.001$; continuation phase: est. $=4.81, \mathrm{SE}=0.70, t=6.91$, $d f=461, P<0.001)$ were associated with smaller reduction in activity impairment. In separate mixed model analyses that excluded baseline WSAS and CPFQ scores, we found that higher baseline activity impairment (acute phase: est. $=0.29$, $\mathrm{SE}=0.03, t=10.30, d f=600, P<0.001$; continuation phase: est. $=0.22, \mathrm{SE}=0.03, t=6.30, d f=454, P<0.001)$, depression severity (acute phase: est. $=0.86, \mathrm{SE}=0.24, t=3.54, d f=609$, $P<0.001$; continuation phase: est. $=0.61, \mathrm{SE}=0.30, t=2.03$, $d f=462, P=0.04)$, and number of general medical conditions (acute phase: est. $=2.94, \mathrm{SE}=0.59, t=4.98, d f=596, P<0.001$; continuation phase: est. $=4.27, \mathrm{SE}=070, t=6.06, d f=455$,

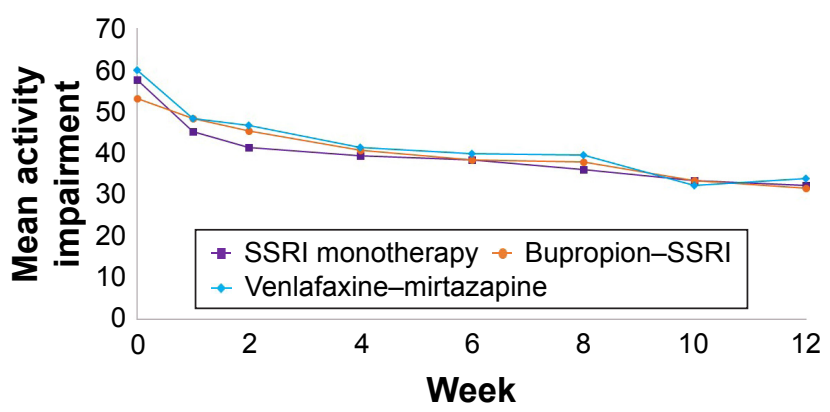

Figure 2 Change in activity impairment during acute phase of CO-MED trial (NCT00590863) based on treatment arm.

Note: The three treatment arms were as follows: escitalopram plus placebo (SSRI monotherapy), sustained-release bupropion plus escitalopram (bupropion-SSRI), and extended-release venlafaxine plus mirtazapine (venlafaxine-mirtazapine).

Abbreviations: CO-MED, Combining Medications to Enhance Depression Outcomes; SSRI, selective serotonin reuptake inhibitor. 
$P<0.001$ ) as well as being unemployed (acute phase: est. $=9.14$, $\mathrm{SE}=1.73, t=5.28, d f=601, P<0.001$; continuation phase: est. $=11.53, \mathrm{SE}=2.10, t=5.48, d f=463, P<0.001)$ and presence of anhedonia (acute phase: est. $=3.85, \mathrm{SE}=1.82, t=2.11, d f=603$, $P<0.035$; continuation phase: est. $=2.71, \mathrm{SE}=2.22, t=1.22$, $d f=456, P=0.22$ ) were associated with smaller reduction in depression severity.

Does activity impairment relate to other clinical outcomes such as symptom severity, function, and side effect burden during course of antidepressant treatment?

Yes. The correlation between levels of activity impairment and psychosocial function were the strongest during both acute (coefficient $=0.68$ ) and continuation ( coefficient $=0.70$ ) phases. Levels of activity impairment also correlated strongly with levels of depressive symptoms, cognitive and physical function, and side effects (Table 2). Among individual depressive symptom domains, energy level, sad mood, and concentration were the three most strongly associated with activity impairment whereas weakest correlations were noted for appetite and suicidal ideations.

Table 2 Correlation coefficients of activity impairment and selfreported depression symptoms, functional outcomes, and side effects

\begin{tabular}{llll}
\hline & Acute phase & Continuation phase \\
\cline { 2 - 2 } Coefficient & & Coefficient \\
\hline WSAS & 0.68 & 0.70 \\
CPFQ & 0.51 & 0.56 \\
QOLI & -0.40 & -0.47 \\
FIBSER & 0.48 & 0.29 \\
QIDS-SR & & \\
Total score & 0.54 & 0.57 \\
Sad mood & 0.46 & 0.45 \\
Sleep & 0.31 & 0.30 \\
Appetite & 0.17 & 0.14 \\
Concentration & 0.43 & 0.43 \\
View of self/guilt & 0.32 & 0.35 \\
Thoughts of death or suicide & 0.25 & 0.24 \\
General interest & 0.39 & 0.44 \\
Energy level & 0.50 & 0.54 \\
Psychomotor agitation/ & 0.35 & 0.36 \\
retardation & & \\
\hline
\end{tabular}

Notes: The nine symptom domains of QIDS-SR are consistent with the diagnostic criteria of major depressive disorder. Participation beyond acute phase (baseline to 3 months) in CO-MED trial (NCT00590863), referred to as continuation phase of an additional 4 months, was restricted to participants with clinical improvement. The correlation coefficients reported here were calculated using repeated observations for each subject during both acute and continuation phases separately.

Abbreviations: WSAS, Work and Social Adjustment Scale; CPFQ, Cognitive and Physical Functioning Questionnaire; QOLI, Quality of Life Inventory; FIBSER, Frequency, Intensity, and Burden of Side Effect Rating; QIDS-SR, Quick Inventory of Depressive Symptomatology, self-report; CO-MED, Combining Medications to Enhance Depression Outcomes.
Does activity impairment level at week 6 predict longer term treatment outcomes even after controlling for remission status and select baseline sociodemographic and clinical variables?

Yes. Participants with no or minimal activity impairment at week 6 were two to three times more likely to be in remission at 3 and 7 months as compared to their counterparts with either moderate or severe activity impairment at week 6 even after controlling for remission status at week 6 and select baseline variables, refer Table 3 for results of univariate and multivariate logistic regression analyses. Additionally, no or minimal activity impairment at week 6 was associated with markedly lower depression severity during rest of acute phase $(F=88.15$, $d f=2,487, P<0.0001)$ and continuation phase $(F=52.53$,

Table 3 Prediction of short- and long-term remission based on activity impairment categories at week 6

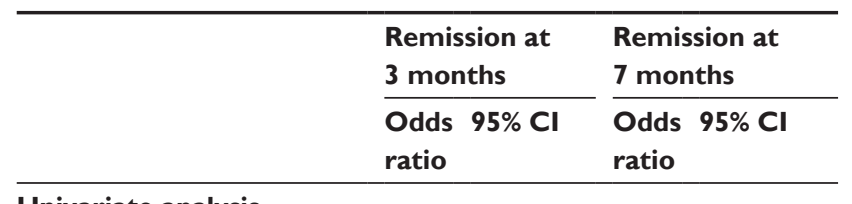

\begin{tabular}{|c|c|c|c|c|}
\hline \multicolumn{5}{|c|}{ Univariate analysis } \\
\hline \multicolumn{5}{|c|}{ Activity impairment category at week 6} \\
\hline No or minimal vs moderate & 2.62 & $1.71,4.02$ & 1.96 & $1.29,2.98$ \\
\hline No or minimal vs severe & 5.29 & $3.14,8.85$ & 4.37 & $2.65,1.79$ \\
\hline \multicolumn{5}{|c|}{ Multivariate analysis with backwards elimination } \\
\hline \multicolumn{5}{|c|}{ Activity impairment category at week 6} \\
\hline No or minimal vs moderate & 1.74 & I.07, 2.84 & 1.61 & $1.002,2.59$ \\
\hline No or minimal vs severe & 2.60 & $1.49,4.83$ & 3.25 & $1.77,5.95$ \\
\hline In remission at week 6 & 3.31 & $2.04,5.39$ & 2.19 & $1.36,3.53$ \\
\hline Female vs male sex & 1.05 & $0.66,1.66$ & 1.22 & $0.79,1.89$ \\
\hline \multicolumn{5}{|l|}{ Treatment arm } \\
\hline $\begin{array}{l}\text { Bupropion-SSRI vs SSRI } \\
\text { monotherapy }\end{array}$ & 1.02 & $0.61,1.69$ & 1.13 & $0.70,1.84$ \\
\hline $\begin{array}{l}\text { Venlafaxine-mirtazapine vs } \\
\text { SSRI monotherapy }\end{array}$ & I.II & $0.67,1.83$ & 1.04 & $0.64,1.69$ \\
\hline Employed at baseline & 1.41 & $0.92,2.17$ & 1.02 & $0.67,1.53$ \\
\hline $\begin{array}{l}\text { Presence of suicidal } \\
\text { ideations at baseline }\end{array}$ & 0.70 & $0.45,1.08$ & 0.52 & $0.34,0.79$ \\
\hline $\begin{array}{l}\text { Presence of anxious features } \\
\text { at baseline }\end{array}$ & 0.99 & $0.61,1.60$ & 1.18 & $0.74,1.87$ \\
\hline $\begin{array}{l}\text { Number of comorbid } \\
\text { medical conditions }\end{array}$ & 1.01 & $0.87,1.17$ & 1.02 & $0.89,1.18$ \\
\hline $\begin{array}{l}\text { Number of comorbid } \\
\text { psychiatric disorders }\end{array}$ & 0.81 & $0.68,0.96$ & 0.85 & $0.73,0.995$ \\
\hline Baseline depression severity & 0.99 & $0.92,1.06$ & 1.00 & $0.93,1.06$ \\
\hline $\begin{array}{l}\text { Baseline cognitive and } \\
\text { physical function }\end{array}$ & 0.96 & $0.92,1.06$ & 0.99 & $0.94,1.03$ \\
\hline Baseline psychosocial function & 1.00 & $0.97,1.03$ & 1.02 & $0.99,1.05$ \\
\hline Baseline quality of life & 0.99 & $0.98,1.01$ & 1.01 & $0.99,1.02$ \\
\hline \multicolumn{5}{|c|}{ Activity impairment category at baseline } \\
\hline No or minimal vs moderate & 1.04 & $0.58,1.86$ & 0.68 & $0.39,1.19$ \\
\hline Severe vs moderate & 1.42 & $0.84,2.39$ & 0.80 & $0.49,1.30$ \\
\hline
\end{tabular}

Notes: Values shown in bold represent statistically significant $(P<0.05)$ findings. Data from the CO-MED trial (NCT00590863).

Abbreviations: $\mathrm{Cl}$, confidence interval; CO-MED, Combining Medications to Enhance Depression Outcomes; SSRI, selective serotonin reuptake inhibitor. 


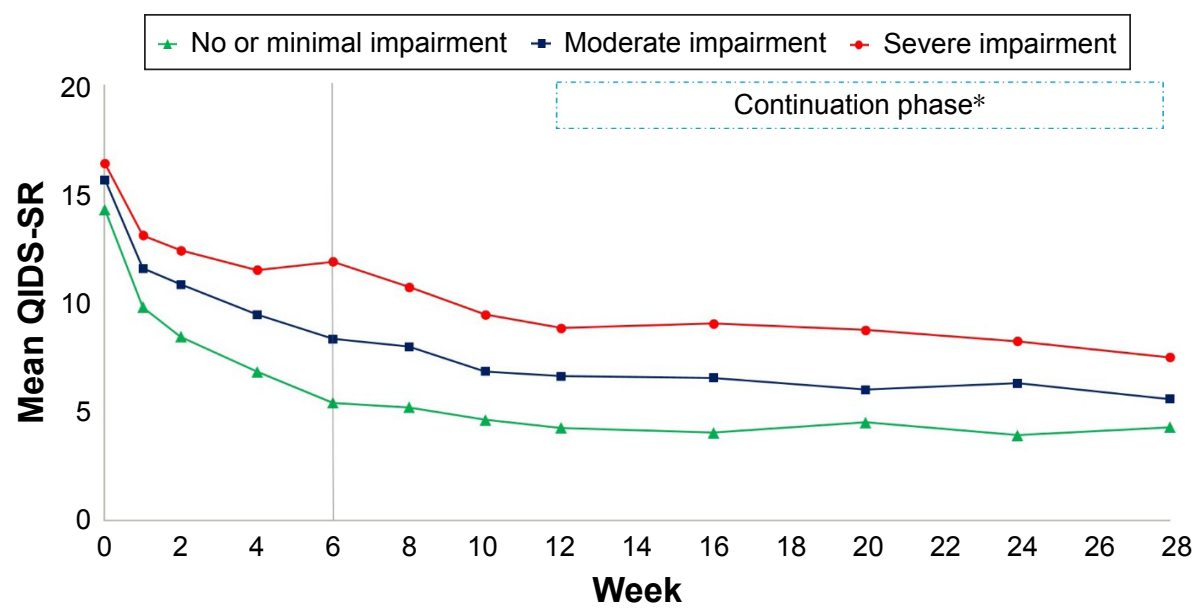

Figure 3 Depression severity levels of depressed outpatients ( $n=665)$ in the CO-MED trial (NCT00590863) based on week 6 activity impairment category. Notes: *Participation in continuation phase was restricted to those participants who experienced clinical improvement during acute phase. Vertical line marks week 6, which was used to define activity impairment categories. No or minimal, moderate, and severe activity impairment were defined as scores of activity impairment $<25.22$, 25.22-62.75, and $>62.75$ based on the mean and standard deviation of activity impairment reported in the 2009 National Health and Wellness Survey. ${ }^{31}$

Abbreviations: CO-MED, Combining Medications to Enhance Depression Outcomes; QIDS-SR, Quick Inventory of Depressive Symptomatology, self-report.

$d f=2,435, P<0.0001$, Figure 3). This difference in depression severity continued to be significant during the rest of acute phase $(F=29.39, d f=2,469, P<0.0001)$ and continuation phase $(F=16.96, d f=2,417, P<0.0001)$ even after controlling for remission status at week 6 and select baseline variables such as sex, treatment arm, employment status, baseline suicidal ideation, depression severity, psychosocial function, cognitive and physical function, quality of life, activity impairment category, presence of anxious features, and number of comorbid medical conditions and psychiatric disorders.

\section{Discussion}

This study found that almost half of depressed outpatients have severe activity impairment prior to treatment initiation, which improves with antidepressant treatment. Monotherapy with SSRI and the combinations of bupropion-SSRI and venlafaxinemirtazapine had similar improvements in activity impairment, which is consistent with the findings previously reported in respect to depression symptom severity in the CO-MED trial. Additionally, after an extensive search of baseline variables, we found that greater impairment in psychosocial function and cognitive function and higher number of general medical conditions were associated with greater likelihood of moderate or severe activity impairment at baseline as well as during the course of antidepressant treatment. In additional analyses that excluded impairments in psychosocial and cognitive function, we found that higher depression severity and being unemployed at baseline were associated with smaller reductions in activity impairment over both acute and continuation phases.

Our findings of improvement in activity impairment with treatment are consistent with those previously reported by
Lam et al. ${ }^{22}$ The strength of correlation between levels of depression severity and activity impairment in our report (correlation coefficient $=0.54$ and 0.57 during acute and continuation phases, respectively) are also comparable to those reported by Lam et $\mathrm{al}^{22}$ (correlation coefficient between activity impairment and HRSD17 =0.56). Previous studies have reported that depressed patients place greater importance to improvement in functioning as compared to reduction in depression severity. ${ }^{44,45}$ This could potentially be explained by the fact that ability to perform day-to-day routine activities correlates more strongly with measures of psychosocial function than depression severity. The findings of our study add to the extant literature that assessing change in depression severity is inadequate in measuring the burden associated with depression ${ }^{15}$ as well as the improvement with antidepressant treatment. ${ }^{16}$

Findings of this study have significant clinical implications. We have found that in depressed patients, persistent activity impairment can be an early marker that can help identify patients who may benefit with augmentation strategies or changes in treatment. Among specific symptom domains of depression, fatigue or lack of energy was most strongly associated with activity impairment. Hence, specific adjunct treatment(s) focused on reducing fatigue and improving energy may reduce activity impairment and ultimately improve longer term clinical outcomes. Specifically, exercise, an efficacious augmentation strategy for depression, ${ }^{46}$ leads to reduced fatigue ${ }^{47,48}$ and can be offered early in course of treatment for patients with persistent activity impairment.

There are several limitations to our report as it is a secondary analysis. Antidepressant medications used in this study 
were not meant to independently improve activity impairment. All treatment arms included active antidepressant medications, and lack of placebo arm is a limitation of study design. Additionally, measure of activity impairment in our report was single-item, subjective, and based on self-report. Hence, it likely differs from objective measures, such as those collected as part of collateral information from relatives, friends, and family members, and does not fully assess impairment in different activities of life. Further, the generalizability of our findings to routine clinical care may be restricted due to high-quality measurement-based care received by participant, even though they were recruited from real-world outpatient clinics with broad inclusion criteria. Measurement-based care, while included in most treatment guidelines for depression, has not yet become part of routine clinical practice.

\section{Conclusion}

We have found that depressed patients have significant impairments in day-to-day routine activities that can be readily measured by a single item that strongly relates to overall psychosocial function. Acute-phase medication treatments improve daily function regardless of the type of medication. Early normalization of activity impairment by week 6 is associated with better long-term clinical outcomes even after controlling for remission status at week 6. Brief systematic assessment of activity impairment during the course of antidepressant treatment can help inform clinical decision-making.

\section{Acknowledgments}

The authors thank the clinical staff at each clinical site including site investigators and coinvestigators for their assistance with this project; all of the study participants; and Savitha Kalidas, $\mathrm{PhD}$, for administrative support. Funded by NIMH under contract N01 MH-90003 to the University of Texas Southwestern Medical Center at Dallas (principal investigators, AJ Rush and MH Trivedi). Forest Pharmaceuticals, GlaxoSmithKline, Organon, and Wyeth Pharmaceuticals provided medications for this trial at no cost. This work was also supported in part through the Center for Depression Research and Clinical Care (principal investigator: Madhukar H Trivedi, MD) and Hersh Foundation (principal investigator: Madhukar H Trivedi, MD). The content of this publication does not necessarily reflect the views or policies of the US Department of Health and Human Services, nor does mention of trade names, commercial products, or organizations imply endorsement by the US government. NIMH had no role in the drafting or review of the manuscript or in the collection or analysis of the data.

\section{Disclosure}

Dr Jha, Dr Minhajuddin, and Mr Teer report no potential conflicts of interest. Dr Greer has received research funding from NARSAD and honoraria and/or consultant fees from H Lundbeck A/S and Takeda Pharmaceuticals International, Inc. Dr Rush has received consulting fees from the American Psychiatric Association, Brain Resource Ltd., H. Eli Lilly, Emmes Corp., Liva-Nova, Lundbeck A/S, Medavante, Inc., Montana State University, National Institute of Drug Abuse, Santium Inc., Takeda USA, speaking fees from the University of California at San Diego, Hershey Penn State Medical Center, the American Society for Clinical Psychopharmacology, the New York State Psychiatric Inst, Stanford Medical School; royalties from Guilford Publications and the University of Texas Southwestern Medical Center; and research support from Duke-National University of Singapore. Dr Trivedi is, or has been, an advisor/consultant for, and received fees from Alkermes, AstraZeneca, Cerecor, Eli Lilly \& Company, Lundbeck, Naurex, Neuronetics, Otsuka Pharmaceuticals, Pamlab, Pfizer Inc., SHIRE Development, and Takeda. In addition, he has received grants/research support from the National Institute of Mental Health and National Institute on Drug Abuse. The authors report no other conflicts of interest in this work.

\section{References}

1. Hasin DS, Goodwin RD, Stinson FS, Grant BF. Epidemiology of major depressive disorder: results from the national epidemiologic survey on alcoholism and related conditions. Arch Gen Psychiatry. 2005; 62(10): 1097-1106.

2. Kessler RC, Berglund P, Demler O, et al; National Comorbidity Survey Replication. The epidemiology of major depressive disorder. JAMA. 2003;289(23):3095-3105.

3. Vos T, Barber RM, Bell B, et al. Global, regional, and national incidence, prevalence, and years lived with disability for 301 acute and chronic diseases and injuries in 188 countries, 1990-2013: a systematic analysis for the Global Burden of Disease Study 2013. Lancet. 2015;386(9995): 743-800.

4. Gelenberg AJ, Freeman MP, Markowitz JC, et al. Practice guideline for the treatment of patients with major depressive disorder third edition. Am J Psychiatry. 2010;167(10):1.

5. Trivedi MH, Morris DW, Wisniewski SR, et al. Increase in work productivity of depressed individuals with improvement in depressive symptom severity. Am J Psychiatry. 2013;170(6):633-641.

6. Beck A, Crain AL, Solberg LI, et al. Severity of depression and magnitude of productivity loss. Ann Fam Med. 2011;9(4):305-311.

7. Trivedi MH, Dunner DL, Kornstein SG, et al. Psychosocial outcomes in patients with recurrent major depressive disorder during 2 years of maintenance treatment with venlafaxine extended release. $J$ Affect Disord. 2010;126(3):420-429.

8. Bech P. Social functioning: should it become an endpoint in trials of antidepressants? CNS Drugs. 2005;19(4):313-324.

9. Ishak WW, Christensen S, Sayer G, et al. Sexual satisfaction and quality of life in major depressive disorder before and after treatment with citalopram in the STAR*D study. J Clin Psychiatry. 2013;74(3):256-261.

10. Jha MK, Minhajuddin A, Thase ME, Jarrett RB. Improvement in self-reported quality of life with cognitive therapy for recurrent major depressive disorder. J Affect Disord. 2014;167:37-43. 
11. Stein MB, Heimberg RG. Well-being and life satisfaction in generalized anxiety disorder: comparison to major depressive disorder in a community sample. J Affect Disord. 2004;79(1-3):161-166.

12. Quilty LC, Mainland BJ, McBride C, Bagby RM. Interpersonal problems and impacts: further evidence for the role of interpersonal functioning in treatment outcome in major depressive disorder. $J$ Affect Disord. 2013;150(2):393-400.

13. Wells KB, Sherbourne CD. Functioning and utility for current health of patients with depression or chronic medical conditions in managed, primary care practices. Arch Gen Psychiatry. 1999;56(10):897-904.

14. Hays RD, Wells KB, Sherbourne CD, Rogers W, Spritzer K. Functioning and well-being outcomes of patients with depression compared with chronic general medical illnesses. Arch Gen Psychiatry. 1995;52(1):11-19.

15. Cohen RM, Greenberg JM, IsHak WW. Incorporating multidimensional patient-reported outcomes of symptom severity, functioning, and quality of life in the Individual Burden of Illness Index for Depression to measure treatment impact and recovery in MDD. JAMA Psychiatry. 2013;70(3):343-350.

16. Jha MK, Greer TL, Grannemann BD, Carmody T, Rush AJ, Trivedi MH. Early normalization of quality of life predicts later remission in depression: findings from the CO-MED trial. J Affect Disord. 2016;206:17-22.

17. Jha MK, Minhajuddin A, GreerTL, Carmody T, Rush AJ, Trivedi MH.Early improvement in psychosocial function predicts longer-term symptomatic remission in depressed patients. PLoS One. 2016;11(12):e0167901.

18. Jha MK, Minhajuddin A, Greer TL, Carmody T, Rush AJ, Trivedi MH. Early improvement in work productivity predicts future clinical course in depressed outpatients: findings from the CO-MED trial. Am $J$ Psychiatry. 2016;173(12):1196-1204.

19. Jakubovski E, Bloch MH. Prognostic subgroups for citalopram response in the STAR*D trial. J Clin Psychiatry. 2014;75(7):738-747.

20. Fujii RK, Goren A, Annunziata K, Mould-Quevedo J. Prevalence, awareness, treatment, and burden of major depressive disorder: estimates from the national health and wellness survey in Brazil. Value Health Regional Issues. 2012;1(2):235-243.

21. Gupta S, Goren A, Dong P, Liu D. Prevalence, awareness, and burden of major depressive disorder in urban China. Expert Rev Pharmacoecon Outcomes Res. 2016;16(3):393-407.

22. Lam RW, Endicott J, Hsu MA, Fayyad R, Guico-Pabia C, Boucher M. Predictors of functional improvement in employed adults with major depressive disorder treated with desvenlafaxine. Int Clin Psychopharmacol. 2014;29(5):239-251.

23. Rush AJ, Kraemer HC, Sackeim HA, et al. Report by the ACNP task force on response and remission in major depressive disorder. Neuropsychopharmacology. 2006;31(9):1841-1853.

24. Rush AJ, Trivedi MH, Stewart JW, et al. Combining medications to enhance depression outcomes (CO-MED): acute and long-term outcomes of a single-blind randomized study. Am J Psychiatry. 2011; 168(7):689-701

25. Vittengl JR, Clark LA, Jarrett RB. Improvement in social-interpersonal functioning after cognitive therapy for recurrent depression. Psychol Med. 2004;34(4):643-658.

26. Trivedi MH, Rush AJ, Wisniewski SR, et al; STAR*D Study Team. Evaluation of outcomes with citalopram for depression using measurement-based care in STAR*D: implications for clinical practice. Am J Psychiatry. 2006;163(1):28-40.

27. Trivedi MH, Rush AJ, Ibrahim HM, et al. The Inventory of Depressive Symptomatology, Clinician Rating (IDS-C) and Self-Report (IDS-SR), and the Quick Inventory of Depressive Symptomatology, Clinician Rating (QIDS-C) and Self-Report (QIDS-SR) in public sector patients with mood disorders: a psychometric evaluation. Psychol Med. 2004; 34(1):73-82.

28. Wisniewski SR, Rush AJ, Balasubramani GK, Trivedi MH, Nierenberg AA, STARD Investigators. Self-rated global measure of the frequency, intensity, and burden of side effects. $J$ Psychiatr Pract. 2006;12(2):71-79.
29. Reilly MC, Zbrozek AS, Dukes EM. The validity and reproducibility of a work productivity and activity impairment instrument. Pharmacoeconomics. 1993;4(5):353-365.

30. Reilly MC [webpage on the Internet]. WPAI Scoring. Available from: http://www.reillyassociates.net/WPAI_Scoring.html. Accessed May 17, 2015.

31. Gupta S, Goren A, Phillips AL, Stewart M. Self-reported burden among caregivers of patients with multiple sclerosis. Int JMS Care. 2012;14(4): 179-187.

32. Rush AJ, Bernstein IH, Trivedi MH, et al. An evaluation of the quick inventory of depressive symptomatology and the Hamilton rating scale for depression: a sequenced treatment alternatives to relieve depression trial report. Biol Psychiatry. 2006;59(6):493-501.

33. Rush AJ, Trivedi MH, Ibrahim HM, et al. The 16-item quick inventory of depressive symptomatology (QIDS), clinician rating (QIDS-C), and self-report (QIDS-SR): a psychometric evaluation in patients with chronic major depression. Biol Psychiatry. 2003;54(5):573-583.

34. Mundt JC, Marks IM, Shear MK, Greist JH. The Work and Social Adjustment Scale: a simple measure of impairment in functioning. Br J Psychiatry. 2002;180:461-464.

35. Zahra D, Qureshi A, Henley W, et al. The work and social adjustment scale: reliability, sensitivity and value. Int J Psychiatry Clin Pract. 2014; 18(2):131-138

36. Frisch M. Quality-of-life-inventory. In: Michalos A, editor. Encyclopedia of Quality of Life and Well-Being Research. Netherlands: Springer; 2014:5374-5377.

37. McAlinden NM, Oei TP. Validation of the quality of life inventory for patients with anxiety and depression. Compr Psychiatry. 2006;47(4): 307-314.

38. Danovitch I, Endicott J. Quality of life measures. In: Rush AJ, First MB, Blacker D, editors. Handbook of Psychiatric Measures. Washington, DC, USA: American Psychiatric Publishing; 2008:125-140.

39. Fava M, Iosifescu DV, Pedrelli P, Baer L. Reliability and validity of the Massachusetts general hospital cognitive and physical functioning questionnaire. Psychother Psychosom. 2009;78(2):91-97.

40. Baer L, Ball S, Sparks J, et al. Further evidence for the reliability and validity of the Massachusetts General Hospital Cognitive and Physical Functioning Questionnaire (CPFQ). Ann Clin Psychiatry. 2014;26(4): 270-280.

41. Schatzberg AF, DeBattista C, Lazzeroni LC, et al. ABCB1 genetic effects on antidepressant outcomes: a report from the iSPOT-D Trial. Am J Psychiatry. 2015;172(8):751-759.

42. Hamlett A, Ryan L, Wolfinger R. On the use of PROC MIXED to estimate correlation in the presence of repeated measures. Proc Statistics and Data Analysis SAS Users Group International. Vol. 19. 2006. Available from: http://www2.sas.com/proceedings/sugi29/198-29.pdf. Accessed November 11, 2016.

43. Bland JM, Altman DG. Statistics notes: correlation, regression, and repeated data. BMJ. 1994;308(6933):896.

44. Zimmerman M, Martinez JA, Attiullah N, et al. Why do some depressed outpatients who are in remission according to the Hamilton Depression Rating Scale not consider themselves to be in remission? J Clin Psychiatry. 2012;73(6):790-795.

45. Zimmerman M, McGlinchey JB, Posternak MA, Friedman M, Attiullah N, Boerescu D. How should remission from depression be defined? The depressed patient's perspective. Am J Psychiatry. 2006; 163(1):148-150.

46. Trivedi MH, Greer TL, Church TS, et al. Exercise as an augmentation treatment for nonremitted major depressive disorder: a randomized, parallel dose comparison. J Clin Psychiatry. 2011;72(5):677-684.

47. Fulcher KY, White PD. Randomised controlled trial of graded exercise in patients with the chronic fatigue syndrome. BMJ. 1997; 314(7095): 1647.

48. Tench CM, McCarthy J, McCurdie I, White PD, D'Cruz DP. Fatigue in systemic lupus erythematosus: a randomized controlled trial of exercise. Rheumatology. 2003;42(9):1050-1054. 
Neuropsychiatric Disease and Treatment

Dovepress

\section{Publish your work in this journal}

Neuropsychiatric Disease and Treatment is an international, peerreviewed journal of clinical therapeutics and pharmacology focusing on concise rapid reporting of clinical or pre-clinical studies on a range of neuropsychiatric and neurological disorders. This journal is indexed on PubMed Central, the 'PsycINFO' database and CAS,

and is the official journal of The International Neuropsychiatric Association (INA). The manuscript management system is completely online and includes a very quick and fair peer-review system, which is all easy to use. Visit http://www.dovepress.com/testimonials.php to read real quotes from published authors.

Submit your manuscript here: http://www.dovepress.com/neuropsychiatric-disease-and-treatment-journal 